\title{
Benefits and Limitations of MALDI-TOF Mass Spectrometry for the Identification of Microorganisms
}

\author{
Jenna Rychert
}

ARUP Laboratories, Salt Lake City, Utah, USA

\section{Article Info}

\section{Article Notes}

Received: April 19, 2019

Accepted: July 2, 2019

\section{*Correspondence:}

Jenna Rychert, ARUP Laboratories, Salt Lake City, Utah, USA; Email: Jennifer.Rychert@ARUPLab.com.

(c) 2019 Rychert J. This article is distributed under the terms of the Creative Commons Attribution 4.0 International License.

Keywords:

Mass spectrometry

MALDI-TOF MS

Bacteria

Yeast

Mycobacteria

Mold

Microbiological Identification

Clinical Laboratory techniques

\begin{abstract}
Matrix-assisted laser desorption-ionization time of flight mass spectrometry (MALDI-TOF MS) is replacing traditional methods for identifying microorganisms in the clinical laboratory. This relatively simple technique overcomes many of the challenges of identifying bacteria and fungi. As the technology has evolved, the expansion of the databases containing spectra of known organisms has allowed for the identification of species with similar phenotypic, genotypic, and biochemical properties that was not previously possible. This has resulted in improvements in clinical care including improving the diagnosis of infections caused by relatively rare species and decreasing the time to diagnosis. In many cases, this leads to a reduction in the time to appropriate therapy and even a decrease in the length of hospital stays. However, it is not without its limitations. Inherent similarities between organisms and a limited number of spectra in the database can lead to poor discrimination between species, as well as misidentifications. These errors occur with relatively low frequency and can typically be overcome with supplemental testing. The adoption of MALDITOF MS in the clinical microbiology laboratory is revolutionizing infectious disease diagnosis and clinical care.
\end{abstract}

\section{Introduction}

Identification of bacteria and fungi by traditional methods can be a time consuming and complex task. Workup of bacteria and yeasts may include assessing colony and gram stain morphology followed by phenotypic and biochemical testing. For fungi, organisms are often distinguished by their characteristic microscopic and macroscopic morphology. In the case of the mycobacteria, DNA probes or other molecular methods are used to identify members of the M. tuberculosis complex, but identification of the non-tuberculous mycobacteria requires the assessment of phenotypic traits, including colony morphology and growth rate. These traditional methods can prolong the time to diagnosis, since the preparation of one or more subcultures is often necessary for a species level identification. Further, the interpretation of phenotypic characteristics is often subjective, requiring significant experience and training for accurate identification. When these traditional methods are unable to identify an organism, sequencing may be performed, but this often results in long turnaround times and adds significant expense. There are now commercially available matrix-assisted laser desorption-ionization time of flight mass spectrometry (MALDI-TOF MS) platforms that are capable of identifying organisms more quickly, more cheaply, and with more specificity than has previously been possible ${ }^{1-5}$.

MALDI-TOF MS is an analytical technique in which particles are ionized, separated according to their mass-to-charge ratio, and 
measured by determining the time it takes for the ions to travel to a detector at the end of a time-of-flight tube. The resulting spectrum, with mass-to-charge values along the $\mathrm{x}$-axis and intensity along the y-axis, is compared to a database of spectra from known organisms. This technology can identify gram-positive, gram-negative, aerobic and anaerobic bacteria as well as mycobacteria, yeast, and molds, typically at the species level, with accuracy as good and often better than traditional methods when compared to sequencing ${ }^{6-9}$. The method is also more reliable than traditional and molecular methods for microorganism identification. Exceptions to this are species not included in the database and species that are inherently similar to one another ${ }^{6,9}$. Due to the small amount of biomass that is required, testing can often be performed from the primary culture, as long as a single well-isolated colony is available. Sample preparation is relatively simple, and analysis of forty or more samples is possible within an hour ${ }^{10}$. Further, a priori knowledge of the type of organism being tested is not required, thus allowing both highly experienced and less experienced microbiologists to perform the testing. This contributes to a reduction in the time to identification by at least one day for most bacteria ${ }^{3,11}$.

Successful identification of microorganisms using MALDI-TOF MS relies heavily on the database containing the spectra of known organisms. It is critical that it includes a sufficient number of isolates for each species, grown under a variety of conditions such that the spectral library for the organism is sufficiently robust to account for the inherent variability expected for any organism ${ }^{12-19}$. Each of the commercially available platforms has a unique set of organisms in its database. As of this writing, the VITEK MS has been FDA cleared for the identification of 332 bacteria and yeasts, 50 mold, and 19 mycobacteria species or species groups representing a total of 1316 species $^{20}$. The MALDI Biotyper has been FDA cleared for the identification of 294 bacteria and 40 yeast species or species groups covering 425 species $^{21,22}$. Additional libraries that have not been cleared by the FDA are also available for the Biotyper, including a mycobacteria library that covers 164 species and a mold library that covers 152 species/species groups. Both platforms also offer the opportunity for the user to add organisms to a research-use-only database. However, given the difficulty of collecting and analyzing a sufficient number of isolates to be able to accurately identify a species for clinical diagnosis, as well as the additional regulatory requirements that come with this, it has been recommended that development of a lab-specific database only be done in larger centralized reference laboratories ${ }^{23,24}$.

In addition to having different databases, the available platforms also differ in the way that they match the spectra from an unknown to the spectra of known organisms ${ }^{25,26}$. Due to these differences in analytical processing, the numeric confidence for a given identification is not directly comparable between the two systems. Regardless of these differences, these platforms are equally accurate, specific,

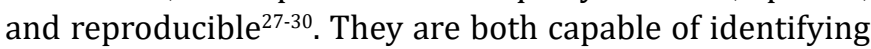
the vast majority of organisms commonly encountered in the clinical laboratory. For those organisms that are not in the database, both systems typically produce a result of 'no identification' rather than an incorrect identification.

Utilization of MALDI-TOF MS in the clinical microbiology lab has markedly increased over the past ten years. Over time, the platforms have gotten progressively better, with significant improvements in the software, interpretive rules, and databases. As a result, there is limited value in comparing results for any category of organisms using a retrospective review of the literature. Therefore, the purpose of this review is to highlight some of the important benefits and limitations of utilizing MALDI-TOF MS for microbial identification in the clinical laboratory.

\section{Benefits}

One of the difficulties that arises when identifying organisms based on traditional or molecular methods is that it can be difficult to discriminate among species that are phenotypically, biochemically, or even genetically similar. Depending on the organism, this may mean that similar species are grouped together (e.g. coagulase negative staphylococci) or an incorrect identification at the species level is provided. MALDI-TOF MS, on the other hand, relies on measuring microbial proteins that are typically well conserved within a species. Thus, it provides a more reliable means of discriminating one species from another. Several studies have shown that MALDI-TOF MS is often able to distinguish between closely related bacterial species with a high degree of confidence ${ }^{31-33}$. This is especially beneficial for organisms in which an incorrect identification or lack of a species level identification could have a significant clinical impact. This could include species that are predictably resistant to specific antibiotics, those that have limited therapeutic options, and those in which clinicians base their therapeutic decisions on identification alone because susceptibility testing is not widely performed.

Among the organisms that are particularly difficult to identify to the species level using traditional methods, but readily identified by MALDI-TOF MS, are the coagulasenegative staphylococci and bacteria with complex nutritional requirements such as the nutritionally variant streptococci and organisms in the HACEK group (Haemophilus, Aggregatibacter (previously Actinobacillus), Cardiobacterium, Eikenella, Kingella). For example, one study found that MALDI-TOF MS correctly identified more than $86 \%$ of HACEK isolates, whereas biochemical testing identified less than $77 \%{ }^{31}$. Similarly, discrimination among species within the $S$. mitis group is particularly poor, even 
when using molecular methods such as DNA probes and sequencing. Given the importance of correctly identifying $S$. pneumonia, this can become problematic. MALDITOF MS, on the other hand, can readily discriminate $S$. pneumonia from non-pneumococcal streptococci within the mitis group with misidentification occurring less than $1 \%$ of the time ${ }^{35,36}$. MALDI-TOF MS has also been shown to be more accurate than biochemical methods for definitive identification of the nutritionally variant streptococci (NVS), including Granulicatella and Abiotrophia species $^{37}$. These fastidious gram-positive cocci, with non-specific colony morphology, are normal flora of the oral cavity but can cause invasive infections including endocarditis. Given that MALDI-TOF MS can be performed directly from blood culture bottles ${ }^{38}$, this method also has the potential to drastically reduce the time to diagnosis of invasive infection for these hard to identify organisms.

The improved performance of MALDI-TOF MS over traditional methods seems to have contributed to an increase in the reporting of some relatively rare species. For example, reporting of skin and peri-prosthetic joint infections due to Staphylococcus lugdunensis, a member of the coagulase-negative staphylococci, appears to have increased due to the use of MALDI-TOF MS ${ }^{39}$. This has also been the case of urinary tract infections caused by Aerococcus urinae and other rare uropathogens ${ }^{40}$. Aerococcus urinae is a catalase negative, Gram-positive cocci that often forms pairs, chains, or triads and looks similar to streptococci not only on gram stain but also when grown on blood-agar. Further, biochemical methods are frequently unable to discriminate Aerococcus species from each other or other Gram-positive bacteria ${ }^{41}$. Given the difficulty in accurately identifying $A$. urinae and the fact that it is a relatively uncommon cause of urinary tract infections, it is not surprising that urinary tract infections caused by $A$. urinae appear to have been under-reported prior to the use of MALDI-TOF MS.

The speed with which MALDI-TOF MS can identify microorganisms helps to quickly guide treatment decisions, which is especially critical when the infecting pathogen is unexpected. As was the case described by von Rotz et al. in which a father and son were admitted to an intensive care unit with symptoms of sepsis and gastroenteritis after a camping trip ${ }^{42}$. Blood cultures obtained at the time of admission were positive for Gram-positive rods that were identified directly from the blood culture bottle within an hour by MALDI-TOF MS as Listeria monocytogenes. Given that L. monocytogenes is a rare foodborne pathogen that typically causes invasive infection only in pregnant women, the immunocompromised, and the elderly, this pathogen was not considered when empiric therapy was started. Upon identification, both patients were switched to appropriate antibiotics and recovered without further incident.
Identification of anaerobes and yeast provide additional examples of the benefits of MALDI-TOF MS in terms of improving the turnaround time. Anaerobic organisms typically have slow doubling times. They are also not very biochemically active and often require a large amount of biomass for definitive identification using biochemical methods. Thus, the length of time it takes to get to a final identification using traditional methods is largely spent on growing the organism, not on performing analytical testing. This can delay the time to diagnosis significantly. For yeasts, faster identification can significantly improve clinical outcomes. In immunocompromised hosts, invasive yeast infections typically caused by $C$. albicans, C. glabrata, C. krusei, and C. parapsilosis, are associated with a high rate of morbidity and mortality and each have unique susceptibility profiles ${ }^{43}$. Thus, as the antifungal susceptibilities are predictable based on the species, timely identification can reduce the time to appropriate empiric therapy. This has been shown to lead to improved clinical care and reduction in the length of hospitalization ${ }^{44,45}$.

A significant cost savings can be achieved by adopting MALDI-TOF MS as the primary method of identification in the clinical laboratory. While the initial cost of the instrument is high, the cost savings on reagents and labor can offset the expenditure within a few years ${ }^{4}$. Reagent savings will vary from one laboratory to another, but for the typical moderate to high volume lab, a savings of greater than $50 \%$ would be expected ${ }^{3,4}$. Given that some species will take longer to identify by MALDI-TOF MS than others, labor savings is likely to be minimal ${ }^{4}$. It is anticipated that as the technology improves and more species are reliably identifiable, additional cost savings may be realized.

\section{Limitations}

For organisms commonly encountered in the clinical laboratory, MALDI-TOF MS can accurately identify most closely related species. However, there are some exceptions. The inability to discriminate between related species can be due to the inherent similarity of the organisms themselves. For example, MALDI-TOF MS is currently unable to differentiate E. coli from Shigella. This is likely because these may not be two species, but actually one, as has been suggested by taxonomists ${ }^{46}$. Despite this, some have suggested that differentiating these organisms by MALDI-TOF MS may be possible ${ }^{47,48}$. Other examples includes members of the $B$. cereus group, B. cepacia complex, B mallei/pseudomallei, Achromobacter species, Citrobacter freundii complex, Enterobacter cloacae complex, Salmonella species ${ }^{49}$, as well as the Mycobacterium tuberculosis complex, Mycobacterium abscessus complex, and the Mycobacterium avium complex ${ }^{50,51}$. For inherently similar organisms, it is common to report to the group, complex or genus level. In cases where differentiation to the species level is clinically necessary, supplemental 
testing should be performed. In the future, the addition of proteomic based approaches to the typical MALDI-TOF MS system may improve the discriminatory power of this method and make it possible to identify organisms at the strain or serotype level ${ }^{52}$.

Another reason similar species may be incorrectly identified is a lack of sufficient spectra in the database. In these cases, it is possible to get an incorrect species-level identification or no identification. For example, one study found that an misidentification was common for similar Trichophyton species ${ }^{53}$. Additionally, misidentification can occur when some members of a species complex are in the database, but others are not. For example, in a study by Body et al., the majority of $M$. mucogenicum isolates were accurately identified, whereas a related organism, M. phocaicum, which was not in the database, was most often misidentified as $M$. mucogenicum ${ }^{51}$. While there are cases where misidentifications such as these do not pose a clinical risk, in other instances there can be significant clinical impact. For example, the inability to discriminate the subspecies of $M$. abscessus, including subspecies abscessus, bolletii, and massiliense, is problematic given that they have different levels of resistance to macrolides ${ }^{51}$. Database updates or user created libraries can usually overcome this problem, as has been the case for some anaerobes, including Bacteroides, Fusobacterium, and Lactobacillus species ${ }^{54}$. Alternatively, using back up methods such as sequencing can be an effective as long as the issue is known.

In conclusion, the introduction of MALDI-TOF MS into the clinical laboratory has brought more timely and accurate identification of microorganisms with subsequent improvement in diagnosis and reduction in the time to appropriate therapy. As these platforms continue to improve and become more widely available, the practice of clinical microbiology will be transformed.

\section{References}

1. Neville SA, Lecordier A, Ziochos $\mathrm{H}$, et al. Utility of matrix-assisted laser desorption ionization-time of flight mass spectrometry following introduction for routine laboratory bacterial identification. J Clin Microbiol. 2011; 49: 2980-4.

2. Seng P, Drancourt M, Gouriet F, et al. Ongoing revolution in bacteriology: Routine identification of bacteria by matrix-assisted laser desorption ionization time-of-flight mass spectrometry. Clin Infect Dis. 2009; 49: 543-51.

3. Tan KE, Ellis BC, Lee R, et al. Prospective evaluation of a matrix-assisted laser desorption ionization-time of flight mass spectrometry system in a hospital clinical microbiology laboratory for identification of bacteria and yeasts: A bench-by-bench study for assessing the impact on time to identification and cost-effectiveness. J Clin Microbiol. 2012; 50: 3301-8.

4. Tran A, Alby K, Kerr A, et al. Cost savings realized by implementation of routine microbiological identification by matrix-assisted laser desorption ionization-time of flight mass spectrometry. J Clin Microbiol. 2015; 53: 2473-9.
5. van Veen SQ Claas EC, Kuijper EJ. High-throughput identification of bacteria and yeast by matrix-assisted laser desorption ionization-time of flight mass spectrometry in conventional medical microbiology laboratories. J Clin Microbiol. 2010; 48: 900-7.

6. Clark AE, Kaleta EJ, Arora A, et al. Matrix-assisted laser desorption ionization-time of flight mass spectrometry: A fundamental shift in the routine practice of clinical microbiology. Clin Microbiol Rev. 2013; 26: 547-603.

7. Patel R. Maldi-tof ms for the diagnosis of infectious diseases. Clin Chem. 2015; 61: 100-11.

8. Dingle TC, Butler-Wu SM. Maldi-tof mass spectrometry for microorganism identification. Clin Lab Med. 2013; 33: 589-609.

9. Croxatto A, Prod'hom G, Greub G. Applications of maldi-tof mass spectrometry in clinical diagnostic microbiology. FEMS Microbiol Rev. 2012; 36: 380-407.

10. Manji R, Bythrow M, Branda JA, et al. Multi-center evaluation of the $\operatorname{vitek}(\mathrm{r}) \mathrm{ms}$ system for mass spectrometric identification of nonenterobacteriaceae gram-negative bacilli. Eur J Clin Microbiol Infect Dis. 2014; 33: 337-46.

11. Ge MC, Kuo AJ, Liu KL, et al. Routine identification of microorganisms by matrix-assisted laser desorption ionization time-of-flight mass spectrometry: Success rate, economic analysis, and clinical outcome. J Microbiol Immunol Infect. 2017; 50: 662-8.

12. Becker PT, de Bel A, Martiny D, et al. Identification of filamentous fungi isolates by maldi-tof mass spectrometry: Clinical evaluation of an extended reference spectra library. Med Mycol. 2014; 52: 826-34.

13. Blosser SJ, Drake SK, Andrasko JL, et al. Multicenter matrix-assisted laser desorption ionization-time of flight mass spectrometry study for identification of clinically relevant nocardia spp. J Clin Microbiol. 2016; 54: 1251-8.

14. Buckwalter SP, Olson SL, Connelly BJ, et al. Evaluation of matrixassisted laser desorption ionization-time of flight mass spectrometry for identification of mycobacterium species, nocardia species, and other aerobic actinomycetes. J Clin Microbiol. 2016; 54: 376-84.

15. Khot PD, Bird BA, Durrant RJ, et al. Identification of nocardia species by matrix-assisted laser desorption ionization-time of flight mass spectrometry. J Clin Microbiol. 2015; 53: 3366-9.

16. Lau AF, Drake SK, Calhoun LB, et al. Development of a clinically comprehensive database and a simple procedure for identification of molds from solid media by matrix-assisted laser desorption ionization-time of flight mass spectrometry. J Clin Microbiol. 2013; 51: 828-34.

17. Normand AC, Cassagne C, Gautier M, et al. Decision criteria for malditof ms-based identification of filamentous fungi using commercial and in-house reference databases. BMC Microbiol. 2017; 17: 25.

18. Schulthess B, Ledermann R, Mouttet F, et al. Use of the bruker maldi biotyper for identification of molds in the clinical mycology laboratory. J Clin Microbiol. 2014 ;52: 2797-803.

19. Theel ES, Hall L, Mandrekar J, et al. Dermatophyte identification using matrix-assisted laser desorption ionization-time of flight mass spectrometry. J Clin Microbiol. 2011; 49: 4067-71.

20. bioMerieux I. VITEK MS 510(k) Premarket Notification (K181412). 2018.

21. Bruker Daltonik GmbH. Maldi Biotyper CA (MBT-CA) System, MBT Smart CA System 510(k) Premarket Notification (K163536). 2017.

22. Bruker Daltonik GmbH. Maldi Biotyper CA System De Novo Premarket Notification (DEN170081). 2017.

23. Mancini N, Burioni R, Sanguinetti M, et al. Risks of "blind" automated identification systems in medical microbiology. J Clin Microbiol. 2013; 51: 3911 . 
24. Westblade LF, Jennemann R, Branda JA, et al. Reply to "risks of 'blind' automated identification systems in medical microbiology". J Clin Microbiol. 2013; 51: 3912.

25. Rychert J, Burnham CA, Bythrow M, et al. Multicenter evaluation of the vitek ms matrix-assisted laser desorption ionization-time of flight mass spectrometry system for identification of gram-positive aerobic bacteria. J Clin Microbiol. 2013; 51: 2225-31.

26. Sauer S, Freiwald A, Maier T, et al. Classification and identification of bacteria by mass spectrometry and computational analysis. PLoS One. 2008; 3: e2843.

27. Cherkaoui A, Hibbs J, Emonet S, et al. Comparison of two matrixassisted laser desorption ionization-time of flight mass spectrometry methods with conventional phenotypic identification for routine identification of bacteria to the species level. J Clin Microbiol. 2010; 48: $1169-75$.

28. Mancini N, De Carolis E, Infurnari L, et al. Comparative evaluation of the bruker biotyper and vitek ms matrix-assisted laser desorption ionization-time of flight (maldi-tof) mass spectrometry systems for identification of yeasts of medical importance. J Clin Microbiol. 2013; 51: 2453-7.

29. Mather CA, Rivera SF, Butler-Wu SM. Comparison of the bruker biotyper and vitek ms matrix-assisted laser desorption ionizationtime of flight mass spectrometry systems for identification of mycobacteria using simplified protein extraction protocols. J Clin Microbiol. 2014; 52: 130-8.

30. Wilen $\mathrm{CB}$, McMullen AR, Burnham CA. Comparison of sample preparation methods, instrumentation platforms, and contemporary commercial databases for identification of clinically relevant mycobacteria by matrix-assisted laser desorption ionization-time of flight mass spectrometry. J Clin Microbiol. 2015; 53: 2308-15.

31. Bizzini A, Jaton K, Romo D, et al. Matrix-assisted laser desorption ionization-time of flight mass spectrometry as an alternative to 16s rrna gene sequencing for identification of difficult-to-identify bacterial strains. J Clin Microbiol. 2011; 49: 693-6.

32. Justesen US, Holm A, Knudsen E, et al. Species identification of clinical isolates of anaerobic bacteria: A comparison of two matrix-assisted laser desorption ionization-time of flight mass spectrometry systems. J Clin Microbiol. 2011; 49: 4314-8.

33. Mellmann A, Cloud J, Maier T, et al. Evaluation of matrix-assisted laser desorption ionization-time-of-flight mass spectrometry in comparison to $16 \mathrm{~s}$ rrna gene sequencing for species identification of nonfermenting bacteria. J Clin Microbiol. 2008; 46: 1946-54.

34. Powell EA, Blecker-Shelly D, Montgomery S, et al. Application of matrix-assisted laser desorption ionization-time of flight mass spectrometry for identification of the fastidious pediatric pathogens aggregatibacter, eikenella, haemophilus, and kingella. J Clin Microbiol. 2013; 51: 3862-4.

35. Branda JA, Markham RP, Garner CD, et al. Performance of the vitek ms v2.0 system in distinguishing streptococcus pneumoniae from nonpneumococcal species of the streptococcus mitis group. J Clin Microbiol. 2013; 51: 3079-82.

36. Harju I, Lange C, Kostrzewa M, et al. Improved differentiation of streptococcus pneumoniae and other s. Mitis group streptococci by maldi biotyper using an improved maldi biotyper database content and a novel result interpretation algorithm. J Clin Microbiol. 2017; 55: 914-22.

37. Ratcliffe P, Fang H, Thidholm E, et al. Comparison of maldi-tof ms and vitek 2 system for laboratory diagnosis of granulicatella and abiotrophia species causing invasive infections. Diagn Microbiol Infect Dis. 2013; 77: 216-9.

38. Martinez RM, Bauerle ER, Fang FC, et al. Evaluation of three rapid diagnostic methods for direct identification of microorganisms in positive blood cultures. J Clin Microbiol. 2014; 52: 2521-9.

39. Szabados F, Anders A, Kaase M, et al. Late periprosthetic joint infection due to staphylococcus lugdunensis identified by matrix-assisted laser desorption/ionisation time of flight mass spectrometry: A case report and review of the literature. Case Rep Med. 2011; 2011: 608919.

40. Hemenway AN, Christensen A, Schriever C. Treatment considerations for potential uropathogens detected by precision microbiological testing. Am J Health Syst Pharm. 2018; 75: 1775-82.

41. Cattoir V, Kobal A, Legrand P. Aerococcus urinae and aerococcus sanguinicola, two frequently misidentified uropathogens. Scand J Infect Dis. 2010; 42: 775-80.

42. von Rotz M, Dierig A, Heininger U, et al. Case report: When two and (1/2) men go camping. BMC Infect Dis. 2017; 17: 102.

43. Pappas PG, Kauffman CA, Andes DR, et al. Clinical practice guideline for the management of candidiasis: 2016 update by the infectious diseases society of america. Clin Infect Dis. 2016; 62: e1-50.

44. Garey KW, Rege M, Pai MP, et al. Time to initiation of fluconazole therapy impacts mortality in patients with candidemia: A multiinstitutional study. Clin Infect Dis. 2006; 43: 25-31.

45. Pincus DH, Orenga S, Chatellier S. Yeast identification--past, present, and future methods. Med Mycol. 2007; 45: 97-121.

46. Pupo GM, Lan R, Reeves PR. Multiple independent origins of shigella clones of escherichia coli and convergent evolution of many of their characteristics. Proc Natl Acad Sci U S A. 2000; 97: 10567-72.

47. Paauw A, Jonker D, Roeselers G, et al. Rapid and reliable discrimination between shigella species and escherichia coli using maldi-tof mass spectrometry. Int J Med Microbiol. 2015; 305: 446-52.

48. Khot PD, Fisher MA. Novel approach for differentiating shigella species and escherichia coli by matrix-assisted laser desorption ionizationtime of flight mass spectrometry. J Clin Microbiol. 2013; 51: 3711-6.

49. CLSI. Methods for the identification of cultured microorganisms using matrix-assisted laser desorption/ionization time-of-flight mass spectrometry. M58 Vol 1st Edition ed Wayne PA Clinical and Laboratory Standards Institute 2017.

50. Neuschlova M, Vladarova M, Kompanikova J, et al. Identification of mycobacterium species by maldi-tof mass spectrometry. Adv Exp Med Biol. 2017; 1021: 37-42.

51. Body BA, Beard MA, Slechta ES, et al. Evaluation of the vitek ms v3.0 matrix-assisted laser desorption ionization-time of flight mass spectrometry system for identification of mycobacterium and nocardia species. J Clin Microbiol. 2018; 56.

52. Karlsson R, Gonzales-Siles L, Boulund F, et al. Proteotyping: Proteomic characterization, classification and identification of microorganisms-a prospectus. Syst Appl Microbiol. 2015; 38: 246-57.

53. Rychert J, Slechta ES, Barker AP, et al. Multicenter evaluation of the vitek ms v3.0 system for the identification of filamentous fungi. J Clin Microbiol. 2018; 56.

54. Garner 0, Mochon A, Branda J, et al. Multi-centre evaluation of mass spectrometric identification of anaerobic bacteria using the vitek(r) ms system. Clin Microbiol Infect. 2014; 20: 335-9. 